\title{
Educação em saúde para adolescentes na escola: importância da atenção primária
}

RESUMO| Objetivo: descrever as ações e a importância da educação em saúde na escola para os adolescentes. Método: Pesquisa descritiva, qualitativa, do tipo revisão integrativa, em julho de 2021, nas bibliotecas eletrônicas: Scientific Electronic Library Online (SciELO) e Google acadêmico. Foram encontradas 10 publicações para fazer parte do corpo de resultados desta pesquisa. As publicações foram organizadas em quadro único, com a ajuda do programa da Microsoft Word. Resultados: Percebeu-se que por mais que as ações de educação em saúde para os adolescentes na escola sejam importantes, existem dificuldades na articulação do cuidado, na implementação das ações e integração entre escola-equipe de saúde. Conclusão: A escola é o lugar mais importante para realizar ações de educação em saúde para os adolescentes, visando unir a equipe de saúde com a equipe escolar, oferecendo um serviço de qualidade e proporcionando momentos ímpares junto ao público juvenil.

Palavras-chaves: Educação; Saúde; Adolescentes; Atenção Primária à Saúde

\begin{abstract}
Objective: to define actions and the importance of health education in schools for adolescents. Method: Descriptive, qualitative research, integrative review type, in July 2021, in electronic libraries: Scientific Electronic Library Online (SciELO) and Academic Google. 10 publications were found to be part of the body of results of this research. The publications were organized in a single frame, with the help of the Microsoft Word program. Results: It was noticed that as important as health education actions for adolescents at school are important, there are difficulties in articulating care, implementing actions and integrating the school-health team. Conclusion: The school is the most important place to carry out health education actions for adolescents, uniting the health team with the school team, offering a quality service and providing unique moments with the youth public. Keywords: Education; Health; Teenagers; Primary Health Care.
\end{abstract}

RESUMEN | Objetivo: definir acciones y la importancia de la educación para la salud en las escuelas para adolescentes. Método: Investigación descriptiva, cualitativa, tipo revisión integradora, en julio de 2021, en bibliotecas electrónicas: Scientific Electronic Library Online (SciELO) y Academic Google. Se encontró que 10 publicaciones forman parte del cuerpo de los resultados de esta investigación. Las publicaciones se organizaron en un solo marco, con la ayuda del programa Microsoft Word. Resultados: Se notó que las acciones importantes de educación en salud para los adolescentes en la escuela son importantes, existen dificultades para articular la atención, implementar acciones e integrar el equipo escolar-salud. Conclusión: La escuela es el lugar más importante para realizar acciones de educación en salud para adolescentes, uniendo al equipo de salud con el equipo escolar, ofreciendo un servicio de calidad y brindando momentos únicos con el público juvenil.

Palabras claves: Educación; Salud; Adolescentes; Primeros auxilios.

\begin{tabular}{l}
\hline Izabelita Félix de Oliveira \\
Enfermeira. UNIFIP. \\
ORCID: 0000-0001-5883
\end{tabular}

\section{Perla Figueredo Carreiro Soares}

Enfermeira. Mestre em Neurociência Cognitiva e Comportamento. Pós-graduada em enfermagem obstétrica. Chefe do Núcleo de Serviços Diagnósticos/SES/PB. ORCID: 0000-0002-0407-685X.

\section{Erlânia Souza Costa}

Enfermeira. Pós-graduada em obstetrícia e neonatologia. Faculdade de Enfermagem Nova Esperança.

ORCID: 0000-0002-5018-0404.

Lúcia Gomes de Souza Silva
Enfermeira. Faculdade Maurício de Nas-
sau. Pós-graduada em Urgência/Emergên-
cia e Unidade de Terapia Intensiva.
ORCID: 0000-0001-6998-435X.

\section{Lúcia Gomes de Souza Silva} ORCID: 0000-0001-6998-435X.

\begin{tabular}{l}
\hline Kátia Cristina Barbosa Ferreira \\
Enfermeira. Universidade Estadual da Pa- \\
raíba. \\
ORCID: 0000-0002-3353-2973.
\end{tabular}

Luciene de Souza Santos Albuquerque Enfermeira. Centro Universitário de João Pessoa.

ORCID: 0000-0001-5119-195X

Recebido em: 15/07/2021

Aprovado em: 30/07/2021

INTRODUÇÃO

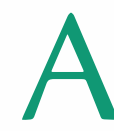
adolescência é uma transição que ocorre na vida humana, e junto a esse processo, acontecem algumas modificações, que perpassam pelas dimensões psicológicas, físicas, emocionais e por vezes familiares $^{(1)}$. Uma das responsabilidades da Atenção Primária à Saúde, é cuidar dos adolescentes, justamente pela vulnerabilidade que este público enfrenta em seu dia-a-dia. Faz parte do compromisso do profissional de saúde, a realização do Programa de Saúde na Escola (PSE) e compreender as mudanças desta fase, além de desenvolver ações de promoção e prevenção em saúde na escola $^{(1)}$.

A escola é uma porta de entrada para o cuidado em saúde dos adolescentes, por ser o local no qual eles pas- 
sam mais tempo durante o seu dia. É na escola, que a equipe multiprofissional precisa estar mais próxima, e buscar atividades de educação em saúde que contemplem aqueles adolescentes da melhor forma possível ${ }^{(2)}$. Entendem-se como educação em saúde, as ações que priorizam a prevenção e promoção da saúde a um determinado público, usando de recursos metodológicos para ensinar, tais como: palestras, jogos interativos, reuniões, ações na comunidade e outros recursos ${ }^{(3)}$.

As temáticas que podem ser trabaIhadas junto aos adolescentes são variadas, podendo abranger: Infecções Sexualmente Transmissíveis (IST's), uso de álcool e outras drogas, conflitos familiares, obesidade, gravidez na adolescência, violência e outros temas imprescindíveis para a saúde do adolescente ${ }^{(4)}$. Dessa forma, compreende-se que promover saúde é pensar e executar de forma educativa, cujo este ato, tem a finalidade de alcançar sujeitos e contextos, possibilitando o direito a saúde e a vida ${ }^{(5)}$. Nas ações realizadas pela atenção primária, a qualidade de vida, redução de agravos, identificação de vulnerabilidades e riscos da população são essenciais para o desenvolvimento do cuidado que será oferecido ${ }^{(5)}$.

Diante desta explanação inicial, percebe-se a importância deste estudo, e a partir disso, construiu-se a seguinte questão de pesquisa: Qual a importância da educação em saúde na escola para os adolescentes realizada pela atenção primária? Tem-se por objetivo: descrever as ações e a importância da educação em saúde na escola para os adolescentes.

\section{MÉTODO}

Pesquisa descritiva, qualitativa, do tipo revisão integrativa. O estudo foi desenvolvido em julho de 2021, nas bibliotecas eletrônicas: Scientific Electronic Library Online (SciELO) e Google acadêmico. Foram elencados

\section{A escola é uma objetivo do estudo. Foram excluídos: Qualquer artigo fora dos critérios de} porta de entrada para o cuidado em saúde dos adolescentes, por ser o local no qual eles passam mais tempo durante o seu dia. É na escola, que a equipe multiprofissional precisa estar mais próxima, e buscar atividades de educação em saúde que contemplem aqueles adolescentes da melhor forma possível os seguintes critérios de inclusão e exclusão para a seleção dos artigos sobre o tema: artigos em inglês, espanhol e português, completos, gratuitos, publicados entre os anos de 2017 a 2021, não duplicados e que respondessem ao inclusão, teses e dissertações.

Foram utilizados os seguintes descritores para a seleção dos artigos: Educação; Saúde; Adolescentes e Atenção Primária à Saúde, cruzados pelo operador booleano AND. Foi utilizado um instrumento, construído pelas autoras deste artigo, apenas para fichamento das publicações selecionadas, para melhor leitura e análise. No fichamento, foram retiradas as seguintes informações: nome dos autores, título, ano de publicação, periódico e os principais achados sobre a educação em saúde para adolescentes na APS.

Realizada a busca, foi possível encontrar 350 publicações inicialmente. A partir do filtro da linha temporal (2017-2021), restaram 98 para análise. As 98 publicações tiveram os títulos lidos, e mediante esta leitura, somente 45 publicações seguiram na análise do estudo. Houve uma segunda leitura dos títulos das 45 publicações, visando averiguar de forma minuciosa se as publicações tinham relação com o tema.

$\mathrm{Na}$ segunda leitura dos títulos, seguiram 30 publicações, pois 15 não correspondiam ao que o objetivo do estudo se referia. Ocorreu a leitura dos resumos das 30 publicações, todos os aspectos contidos nos resumos foram analisados, tais como: introdução, objetivo, método, resultados, discussão e conclusões de cada publicação. A partir desta leitura, foram selecionadas 8 publicações para fazer parte do corpo de resultados desta pesquisa. Houve o auxílio de dois pesquisadores apropriados sobre o tema, no processo de busca.

Por fim, as publicações foram organizadas em quadro único, com a ajuda 
do programa da Microsoft Word.

\section{RESULTADOS}

Abaixo, o quadro apresenta a descrição das publicações encontradas sobre a temática. As publicações trouxeram grande contribuição para este estudo, abordaram sobre os principais pontos relacionados a educação em saúde para adolescentes que acontece na escola, e que é realizada pela APS, que tem por função ofertar a prevenção e promoção a saúde para todos os públicos em seu território, incluindo as escolas.
Houve predominância de publicações no ano de $2020^{(3)}$ e no ano de $2018^{(2)}$. A revista da Escola de Enfermagem da USP foi a que mais publicou no período de 2017 a 2021, conforme as publicações analisadas.

A APS precisa desenvolver várias ações de prevenção e promoção para a saúde dos adolescentes, por intermédio do Programa de Saúde na Escola (PSE), e nas publicações estudadas, foi visto que existem alguns desafios, e que por mais que as ações de educação em saúde para os adolescentes na escola sejam importantes, as dificuldades na articulação do cuidado, na imple- mentação das ações e integração entre escola-equipe de saúde por vezes são fragilizadas, deixando as ações pouco consistentes.

Ficou evidenciado, que o enfermeiro é uma das figuras importantes no cuidado aos adolescentes na escola, sendo o profissional mais relatado nas publicações. Os temas trabalhados nas publicações foram diversos, tais como: obesidade, sexualidade, gestação na adolescência, uso de drogas e álcool, contexto familiar e outros temas pertinentes, além disso, estes temas foram discutidos no tópico abaixo.

\section{QUADRO 1- Descrição das publicações sobre a educação em saúde para adolescentes na APS:}

\begin{tabular}{|c|c|c|c|c|}
\hline Autores & Título & $\begin{array}{c}\text { Ano de } \\
\text { publicação }\end{array}$ & Periódico & $\begin{array}{c}\text { Educação em saúde para adolescentes } \\
\text { na escola realizada pela APS }\end{array}$ \\
\hline \multirow{3}{*}{ Corrêa HW, Toassi RFC.(5) } & \multirow{3}{*}{$\begin{array}{l}\text { Programa Saúde na } \\
\text { Escola: potencialidades } \\
\text { e desafios na construção } \\
\text { de redes de cuidado. }\end{array}$} & \multirow{3}{*}{2018} & \multirow{3}{*}{ Saúde em Redes. } & Pouca resolutividade da equipe de saúde \\
\hline & & & & $\begin{array}{l}\text { Desafios para realizar as ações de saúde para } \\
\text { os adolescentes. }\end{array}$ \\
\hline & & & & $\begin{array}{l}\text { Existem potencialidades nas ações de } \\
\text { educação em saúde na escola }\end{array}$ \\
\hline
\end{tabular}

Assis PICM, Rosa GS, Cecílio AG.(6) $\begin{gathered}\text { Saúde, sexualidade e } \\ \text { adolescência: relato de } \\ \text { uma ação de Educação } \\ \text { em Saúde. }\end{gathered} \quad 2020 . \quad$ Revista de APS.

As atividades desenvolvidas abordavam: uso de preservativos, métodos contraceptivos, infecções sexualmente transmissíveis e gravidez na adolescência.

\begin{tabular}{|c|c|c|c|c|}
\hline & & & & $\begin{array}{c}\text { Existe limitações quanto às práticas } \\
\text { alimentares }\end{array}$ \\
\hline $\begin{array}{c}\text { Diniz CBC, Feitosa AA, Coutinho } \\
\text { BLM, Gomes SC, Sant'anna AL, } \\
\text { Araújo AF, et al.(7) }\end{array}$ & $\begin{array}{l}\text { Acompanhamento nu- } \\
\text { tricional de adolescentes } \\
\text { no Programa Saúde na } \\
\text { Escola. }\end{array}$ & 2020. & J Hum Growth. & $\begin{array}{l}\text { Ênfase na prática preventiva, esquecendo de } \\
\text { questionar as necessidades dos adolescentes. } \\
\text { O acompanhamento nutricional pode } \\
\text { prevenir situações relacionadas a obesidade } \\
\text { e outros questionamentos de acordo com as } \\
\text { necessidades identificadas. }\end{array}$ \\
\hline Vieira CENK. et al.(8) & $\begin{array}{l}\text { Programa de Enferma- } \\
\text { gem Saúde na Escola: } \\
\text { prevenção e controle de } \\
\text { sobrepeso/obesidade } \\
\text { em adolescentes. }\end{array}$ & 2018. & $\begin{array}{l}\text { Revista da Escola de } \\
\text { Enfermagem da USP. }\end{array}$ & $\begin{array}{l}\text { Realização de um mapeamento das ações do } \\
\text { PSE para prevenção da obesidade. } \\
\text { Implantação de intervenções e estratégias } \\
\text { sobre obesidade. }\end{array}$ \\
\hline
\end{tabular}




\begin{tabular}{|c|c|c|c|c|}
\hline & & & & $\begin{array}{l}\text { Dificuldades nas práticas do PSE e na } \\
\text { implantação das ações. }\end{array}$ \\
\hline Brasil EGM. et al.(9) & $\begin{array}{l}\text { Promoção da saúde } \\
\text { de adolescentes e } \\
\text { Programa Saúde na } \\
\text { Escola: complexidade } \\
\text { na articulação saúde e } \\
\text { educação. }\end{array}$ & 2017. & $\begin{array}{l}\text { Revista da Escola de } \\
\text { Enfermagem da USP. }\end{array}$ & $\begin{array}{l}\text { O desconhecimento dos profissionais sobre } \\
\text { o programa. } \\
\text { Falta de planejamento das ações } \\
\text { Desarticulação do cuidado em saúde para os } \\
\text { adolescentes. }\end{array}$ \\
\hline Ramos LS. et al.(10) & $\begin{array}{l}\text { A saúde na escola como } \\
\text { meio de prevenção da } \\
\text { gravidez na adolescên- } \\
\text { cia: uma breve análise. }\end{array}$ & 2020. & $\begin{array}{l}\text { Revista Eletrônica } \\
\text { Acervo Saúde. }\end{array}$ & $\begin{array}{l}\text { Existe uma maior disponibilidade das } \\
\text { famílias para educar-se sobre a gravidez na } \\
\text { adolescência. } \\
\text { O conservadorismo é um entrave para o } \\
\text { cuidado em saúde dos adolescentes }\end{array}$ \\
\hline Silva AA de. et al.(11) & $\begin{array}{l}\text { Ações de promoção } \\
\text { da saúde no Programa } \\
\text { Saúde na Escola no } \\
\text { Ceará: contribuições da } \\
\text { enfermagem. }\end{array}$ & 2021 & $\begin{array}{l}\text { Revista Brasileira de } \\
\text { Enfermagem }\end{array}$ & $\begin{array}{l}\text { Houve aumento da avaliação clínica }(78,7 \% \\
\qquad \text { e } 91,3 \%) \text {. } \\
\text { Promoção da saúde e prevenção de doenças } \\
\qquad(82,5 \% \text { e } 89,3 \%) \text {. } \\
\text { Levantamento de escolares para acompanha- } \\
\text { mento (41,4\% e } 66,4 \%) \text { nas escolas. }\end{array}$ \\
\hline
\end{tabular}

Fonte: dados da pesquisa, 2021.

\section{DISCUSSÃO}

A educação em saúde para adolescentes na escola é uma ação primordial para o cuidado. Um dos temas abordados nas publicações foram as IST's. Sabe-se que existem diversas IST's a serem prevenidas, e na escola, palestras educativas, tanto com os meninos, quanto com as meninas, tornam-se relevantes. Trabalhar a educação sexual é algo imprescindível a ser realizado pela equipe de saúde na escola $a^{(6,9,10)}$. Os temas como IST's, gravidez na adolescência e uso de preservativo são os mais ascendentes nas publicações voltadas à saúde do adolescente. As IST's que frequentemente acometem a população juvenil são: Papiloma Vírus Humano (HPV), hepatite B, Sífilis, Síndrome da Imunodeficiência Humana (AIDS) e Herpes ${ }^{(6,9,10)}$.

$\mathrm{O}$ incentivo para utilizar o preser- vativo nas relações sexuais é importante, por ser um dos métodos mais eficazes não somente na prevenção de gravidez, mas em IST's também. Diante da vulnerabilidade que os adolescentes enfrentam, é preciso orientar quanto ao sexo com mais de um parceiro, inicio de vida sexual precoce ausência de preservativos no ato sexual, e outras problemáticas que podem gerar resultados difíceis na vida do adolescente ${ }^{(6,9,10)}$. Um dos cuidados importantes que precisam ser incentivados para o adolescente, é a vacinação. O calendário vacinal do adolescente é disponível integralmente pelo Sistema Único de Saúde (SUS), fez-se pertinente que a equipe de saúde realizasse a vacinação nas escolas para este público ${ }^{(12) .}$

Em outra publicação analisada, os cuidados com a alimentação, sobrepeso e a obesidade na infância e na adolescência foram elencados como intervenções necessárias na escola, no qual a equipe de saúde pode realizar atividades que auxiliem na melhora da nutrição na adolescência, bem como na prevenção do aumento de peso ${ }^{(7,8)}$. Nesta pesquisa, o enfermeiro esteve presente de forma predominante nas atividades voltadas à alimentação na adolescência, porém, com algumas dificuldades na tomada de decisão sobre o tema. É preciso o engajamento da equipe multiprofissional, no qual o apoio do nutricionista é pertinente. O artigo aborda sobre o Mapeamento de Intervenção, que é um método usado para a construção de programas de promoção em saúde, e neste artigo estava relacionado sobre a obesidade ${ }^{(8)}$.

Neste artigo, abordou-se sobre o início precoce do ato sexual, que por vezes, na adolescência, é realizado sem os devidos cuidados e orientações. Um dos temas necessários é a saúde 
sexual, pois a partir dela, previne-se a gravidez na adolescência. Sabe-se que a gestação é um momento singular, e que o adolescente está em fase de mudanças, aprendizado e almejando diversos objetivos, no qual uma gestação pode dificultar os seus planos para o futuro. Neste contexto, insere-se a temática dos métodos contraceptivos, que fazem parte das orientações de educação em saúde ${ }^{(6,10)}$. É preciso romper com o "tabu" que por diversas vezes atrapalham a aquisição de conhecimentos dos adolescentes, e um deles é como prevenir-se de uma gestação não planejada. A equipe de saúde pode abordar sobre os principais métodos, utilizando de recursos para demonstrar e esclarecer dúvidas deste público ${ }^{(6,10)}$.

Ainda pode ser abordado outros temas na educação em saúde na escola, como por exemplo, o uso de álcool e drogas. Explicar sobre a vulnerabilidade social que os adolescentes vivem, compreender a sua realidade e o acesso que eles possuem ao álcool e outras drogas é relevante, pois a partir disso, pode-se elaborar ações de cuidado em saúde sobre o tema ${ }^{(13)}$. Percebeu-se que existem algumas fragilidades e dificuldades para realizar as ações, porém, também ficou evidenciado que a saúde do adolescente necessita de diversos olhares e saberes intercalados, para propiciar o cuidado ${ }^{(9,11,5)}$.

\section{CONCLUSÃO}

Sugerimos na conclusão responder as questões norteadoras, a relevância do estudo e a inserção da enfermagem neste contexto

A importância da educação em saúde na escola para os adolescentes realizada pela atenção primária, foi vista em todas as publicações, mediante a realização de ações com temas importantes para a fase adolescente, que atuavam diretamente na prevenção e promoção a saúde deste público. O objetivo desta pesquisa foi alcançado, e o estudo abordou as principais formas de educação em saúde na escola para adolescentes, sendo uma temática importante, por tratar-se de um problema de saúde pública pertinente e amplo. Compreendeu-se, que a equipe multiprofissional é primordial, por trazer a perspectiva de cuidar dos adolescentes sob a forma interdisciplinar, abrangendo vários temas que eles precisam em seu dia-a-dia.
Dentre os principais resultados, o enfermeiro teve destaque, e maior proximidade com as ações desenvolvidas na escola. A enfermagem foi uma profissão citada pelas publicações, no qual exerce efetividade em seus cuidados em saúde na escola, criando vínculo com os estudantes e a equipe escolar, desenvolvendo os temas relacionados a saúde do adolescente, sendo uma categoria de grande importância para a realização do saúde na escola.

A escola é o lugar mais importante para realizar ações de educação em saúde para os adolescentes, visando unir a equipe de saúde com a equipe escolar, oferecendo um serviço de qualidade e proporcionando momentos ímpares junto ao público juvenil. A prevenção em saúde é uma importante ferramenta para o cuidado aos adolescentes, por isso, precisa-se incentivar que elas aconteçam de forma contínua e articulada. Recomenda-se novos estudos sobre o tema, pelo fato de que, são temáticas que não cessam na vida dos adolescentes, sendo importante a publicação de novos artigos sobre cada assunto abordado aqui nesta pesquisa.

\section{Referências}

1- Zappe JG; Dell'aglio DD. Adolescência em diferentes contextos de desenvolvimento: risco e proteção em uma perspectiva longitudinal. Revista Psico, vol. 47, núm.2, p 99-110, 2016.

2- Jacóe NB. et al. 0 olhar dos profissionais de uma unidade básica de saúde sobre a implantação do programa saúde na escola. Rev Med. Vol. 24, núm. 1, p 43-48, 2014.

3- Miccas FL, Batista SHS da. Educação permanente em saúde: metassíntese. Rev Saúde Pública vol. 48, núm.1, p170-185, 2014.

4- Carneiro RF. et al. Educação sexual na adolescência: uma abordagem no contexto escolar. Revistas de políticas públicas sanare, sobral, v.14, n.01, p.104-108, 2015.

5- Corrêa HW, Toassi RFC. Programa Saúde na Escola: potencialidades e desafios na construção de redes de cuidado. Saúde em Redes. v.4, n.3, 2018.

6- Assis PICM, Rosa GS, Cecílio AG. Saúde, sexualidade e adolescência: relato de uma ação de Educação em Saúde. Revista de APS. v. 23, 2020.

7- Diniz CBC, Feitosa AA, Coutinho BLM, Gomes SC, Sant'anna AL, Araújo $A F$, et al. Acompanhamento nutricional de adolescentes no Programa
Saúde na Escola. J Hum Growth. 2020. vol.30, n.1, pp. 32-39.

8- Vieira CENK. et al. Programa de Enfermagem Saúde na Escola: prevenção e controle de sobrepeso/obesidade em adolescentes. Revista da Escola de Enfermagem da USP. 2018.

9- Brasil EGM. et al. Promoção da saúde de adolescentes e Programa Saúde na Escola: complexidade na articulação saúde e educação. Revista da Escola de Enfermagem da USP. v. 51, 2017.

10-Ramos LS. et al. A saúde na escola como meio de prevenção da gravidez na adolescência: uma breve análise. Revista Eletrônica Acervo Saúde. 2020.

11- Silva AA de. et al. Ações de promoção da saúde no Programa Saúde na Escola no Ceará: contribuições da enfermagem. Revista Brasileira de Enfermagem. v.74, n.1, 2021.

12- Zardo GP. et al. Vacina como agente de imunização contra o HPV. Ciência \& Saúde Coletiva, vol.19, núm.9, p :3799-3808, 2014.

13- Portela ALB, Garça LF, Roberto JG. Adolescência vulnerável: fatores biopsicossociais relacionados ao uso de drogas. Revista Bioética, vol. 23, núm. 2015. 